\title{
Expulsions following 1000 GyneFix insertions
}

Joanne Dennis, MRCGP, MFF, Career Grade Trainee; Anne Webb, MRCGP, MFFP, MRCOG, Consultant; Meera Kishen, MD, DGO, Dip.Ven., MFF, Consultant, Abacus Centres for Contraception and Reproductive Health, North Mersey Community NHS Trust, Liverpool, $U K$.

Correspondence: Dr Jo Dennis, Central Abacus, 40-46 Dale St, Liverpool, L2 5SF. Tel: 01512842500 , Fax: 01512932005.

(Accepted $11^{\text {th }}$ May 2001)

The Journal of Family Planning and Reproductive Health Care 2001: 27(3): 135-138

\begin{abstract}
Context. The GyneFix intra-uterine device has been used in our family planning service since 1997. One of the perceived advantages is its low expulsion rate, as reported by clinical trials.

Objective. To calculate expulsion rates in routine clinical use and to look at possible reasons for expulsion.

Design. Retrospective casenote analysis and opportunistic client consultation.

Setting. A city centre family planning clinic.

Participants. The first 1000 GyneFix insertions.

Main outcome measures. Parity of client, experience of clinician carrying out insertion, time from device insertion to expulsion.

Results. Overall expulsion rate was $7.6 \%$. There was no significant difference in parity of clients experiencing expulsion. Most (4.7\%) expulsions were early, occurring within 3 months of insertion. There was considerable variation in early expulsion rate from one clinician to another. Later expulsions also occurred, up to 28 months after insertion. Increasing experience of the inserting clinician led to lower rates of late expulsion. Unnoticed expulsion led to four unplanned pregnancies.

Conclusions: The GyneFix expulsion rate in our service is higher than quoted in clinical trials. Early expulsions may be related to insertion technique, representing insufficient implantation of the anchoring knot into the fundal myometrium. Late expulsions also occur, often many months after insertion; the reason for these is unclear. Users should be taught to check for the presence of the thread after each menstrual period and unnoticed expulsion should be confirmed by ultrasound and abdomino-pelvic plain $X$-ray.
\end{abstract}

\section{Key message points}

- Expulsion rate in our service has been found to be higher than quoted in clinical trials.

- Most expulsions occur within 3 months of insertion and may be due to insufficient implantation of the anchoring knot.

- Late expulsions also occur, often many months after insertion. The reason for late expulsions is unclear.

- As a routine, users should be taught to check for the presence of the thread after each menstrual period.

- Unnoticed expulsion should be confirmed by ultrasound and abdomino-pelvic plain X-ray.

\section{Introduction}

The frameless GyneFix intra-uterine device (IUD) has been available in the UK since 1997. It was developed to attempt to overcome side effects seen with framed IUDs such as expulsion, bleeding and pain, thought to be due to incompatibility between the uterine cavity and the frame of the device, particularly in nulliparous women. Clinical trials have confirmed that the GyneFix is effective and acceptable to nulliparous as well as parous women, with low expulsion rates, ${ }^{1}$ although to date there has not been a randomised trial comparing GyneFix to framed IUDs in nulliparous women.

Two randomised comparative studies using the Flexigard, an earlier model of the GyneFix (identical in all but insertion instrument), found the frameless device to have significantly higher rates of expulsion than the framed device, the TCu380A, in parous women..$^{2,3}$ This was found to be due to shortcomings in the insertion instrument, which was subsequently modified, the resultant device being named 'GyneFix'.

Studies using the GyneFix have been more encouraging. The 3-year expulsion rate in a non-comparative trial involving both nulliparous and parous women was $0.7 \% .^{1} \mathrm{~A}$ randomised comparative trial in parous women in China found the expulsion rate with the GyneFix to be significantly lower $(3.0 \%)$ than that of the framed device, the T380A (7.4\%), at 3 years. ${ }^{4}$ The authors state that most expulsions with GyneFix occur within 3 months of insertion, representing 'insertion failures' due to insufficient implantation of the anchoring knot into the fundal myometrium through lack of experience with the technique. A recent paper reported no expulsions in a noncomparative study using the device immediately postabortion in 175 Chinese women, mostly parous. ${ }^{5}$ It is not stated which model of GyneFix, the IN (interval) or PT (post-termination) version, was used.

Liverpool was the first clinical site in the UK to routinely offer GyneFix and as such it is a continuing training centre. All insertions are carried out at our city-centre clinic. A protocol has been drawn up in our service to help clinicians decide who might benefit from using a GyneFix over a framed copper device. The GyneFix is available to all women considering IUD use, but is offered mainly to nulliparous women and those who have experienced IUD expulsion or IUD-related pain in the past. It is used as emergency contraception where ongoing use is intended. The GyneFix IN (interval) is the version used in our service.

A survey carried out amongst the first 215 insertions showed high levels of user satisfaction, $86 \%$ saying that they would recommend the device to a friend. ${ }^{6}$ Some negative comments related to heavier menstrual flow and removals for medical reasons are mostly due to bleeding. However, expulsion rates were higher than quoted in trials. A study of expulsions amongst the first 1000 insertions was therefore undertaken.

\section{Method}

Since insertions began, a register of all GyneFix users has 
been kept. Onto this is entered insertion date, parity (nulliparous meaning never pregnant or no pregnancy over 24 weeks), experience of the clinician carrying out insertion and subsequent events at follow-up, such as removal and expulsion. Clients are advised to re-attend in the event of problems. Clinic staff are regularly reminded to inform the first author of any removals, expulsions or other adverse events, so that these can be noted in the register and kept under review. Data were collected from both the register and by casenote review. Where a client underwent more than one GyneFix insertion, for example if she opted for re-insertion following expulsion, this was counted as two separate insertions and entered onto the register twice.

The clinician carrying out the insertion was classed as 'inexperienced' or 'experienced', depending on the total number of insertions he or she had carried out. The clinician is considered experienced in GyneFix insertion once he or she has inserted more than 10 devices.

Time in months from insertion to expulsion was noted so that expulsions could be classed as 'early', occurring within 3 months of insertion, or 'late', more than 3 months after insertion.

\section{Results}

One thousand devices were successfully inserted from February 1997 to April 2000, by 74 inexperienced and 10 experienced clinicians. Most (799) clients were nulliparous. The remainder (201) were parous.

There were 11 abandoned insertions, either through pain or inability to pass a uterine sound, or failure to anchor the device at the uterine fundus during insertion. Four of these subsequently underwent successful insertion. Only successful insertions have been included in the analysis.

Seventy-six devices are known to have been expelled. There may be further expulsions about which we are unaware, as follow-up is opportunistic. Data were collected up until August 2000. Figure 1 shows time from insertion to expulsion.

Most of the expulsions (47) were early, occurring within 3 months of insertion. The remaining 29 were late, occurring more than 3 months after insertion. Of these, 14 were between four and 6 months, eight between seven and 12 months and seven later than 12 months after insertion. The latest expulsion was 28 months after insertion.

Table 1 Number of insertions and expulsions according to parity

\begin{tabular}{llll}
\hline & Nulliparous $(\%)$ & Parous $(\%)$ & Total $(\%)$ \\
\hline Insertions & $799(80)$ & $201(20)$ & $1000(100)$ \\
Expulsions & $64(8.0)$ & $12(6.0)$ & $76(7.6)$ \\
\hline
\end{tabular}

Table 1 shows the numbers of expulsions according to parity. The expulsion rate amongst nulliparous users was higher $(8.0 \%$ vs. $6.0 \%)$, although this difference does not reach significance using chi-square testing.

\section{Experience of clinician carrying out insertion}

Three hundred and sixty-eight of the 1000 insertions were carried out by an inexperienced clinician (Table 2). Thirtytwo of these $(8.6 \%)$ were subsequently expelled. Forty-four $(7.0 \%)$ of the remaining 632 devices inserted by experienced clinicians were expelled. This difference is not significant using chi-square testing.

\section{Early and late expulsions}

There was no significant difference in early expulsion rate between inexperienced and experienced clinicians using chi-square testing. The late expulsion rate was higher amongst inexperienced clinicians than experienced. This difference is significant using chi-square testing $(\mathrm{p}<0.05)$.

Within the group of experienced clinicians, early expulsion rate ranged from zero to $14.3 \%$, median $1.6 \%$. For late expulsions, although actual numbers are small (between zero and four per experienced clinician), interindividual expulsion rates did not vary to the same degree.

There were four pregnancies through unnoticed expulsion, two at 5 months, one at 13 and one at 20 months from insertion. Five women experienced two GyneFix expulsions (therefore appearing twice in Figure 1).

Figure 1 Number of GyneFix devices expelled, showing time in months from insertion

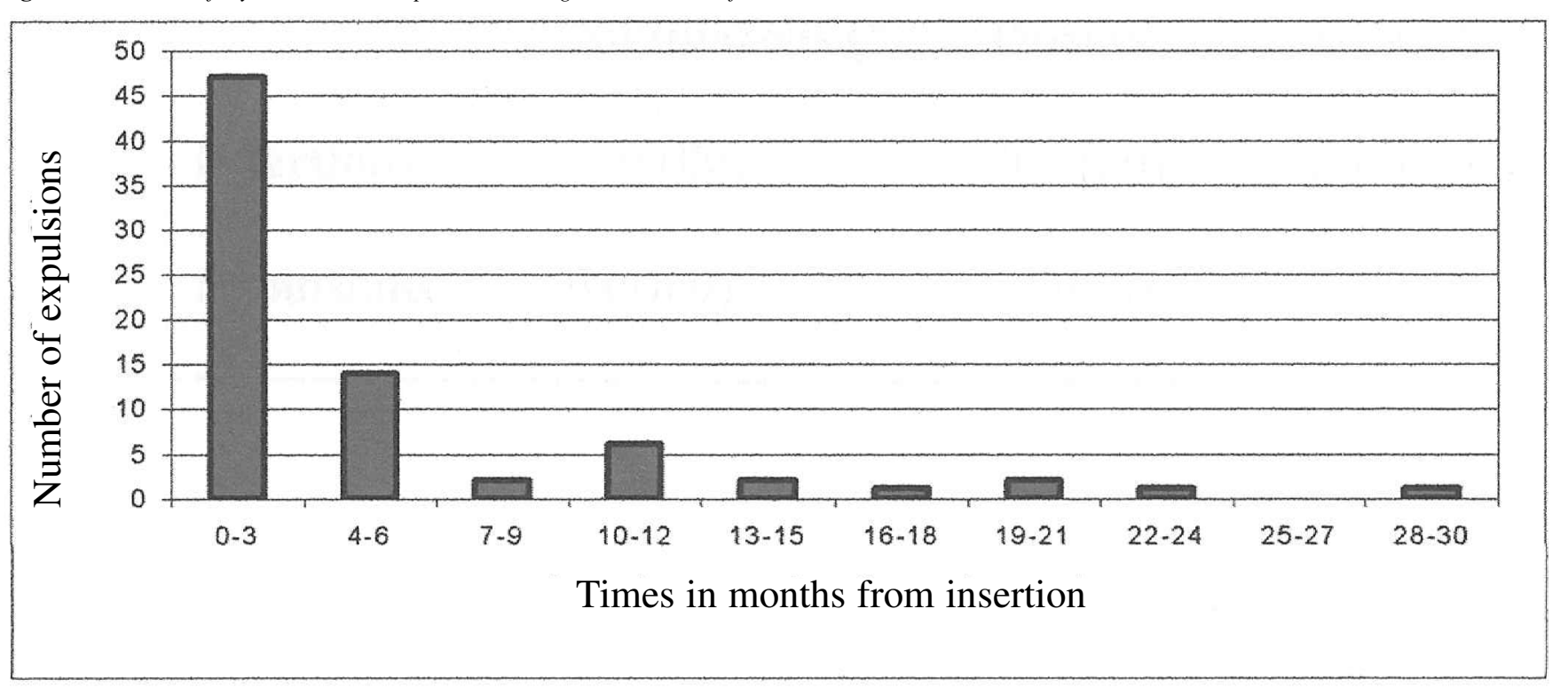


Table 2 Early and late GyneFix expulsion rates for inexperienced and experienced clinicians

\begin{tabular}{|c|c|c|c|c|c|c|}
\hline & \multicolumn{2}{|c|}{ Inexperienced } & \multicolumn{2}{|c|}{ Experienced } & \multicolumn{2}{|c|}{ Total } \\
\hline & Number & $\%$ (range) & Number & $\%$ (range) & Number & $\%$ \\
\hline Total inserted & 368 & 100 & 632 & 100 & 1000 & 100 \\
\hline Early expulsions & 16 & $4.3(0-66.7)$ & 31 & $4.9(0-14.3)$ & 47 & 4.7 \\
\hline Late expulsions & 16 & $4.3(0-33.3)$ & 13 & $2.1(0-4.4)$ & 29 & 2.9 \\
\hline Total expulsions & 32 & 8.6 & 44 & 7.0 & 76 & 7.6 \\
\hline
\end{tabular}

\section{Discussion}

The low rates of GyneFix expulsion seen in clinical trials have not been reproduced in routine use in our clinic setting. Expulsion rates may be higher than presented; incomplete follow-up may have been a source of bias if further expulsions occurred but were not reported.

During insertion, the anchoring knot is embedded into the fundal myometrium to a depth of $9 \mathrm{~mm}$, so that the uppermost copper sleeve is in contact with the endometrial surface of the fundus. If the knot is inadequately embedded, then the device may not be sufficiently anchored for retention, resulting in expulsion. This may be the mechanism for early expulsions. The relatively high rate of early expulsions for experienced clinicians is skewed by considerable variation between clinicians (median early expulsion rate $1.6 \%$, range $0-14.3 \%$ ). Thus early expulsion appears to be operator-dependant.

Later expulsions, occurring more than 3 months after insertion, are more difficult to explain. Inadequate insertion is unlikely to be the sole reason, especially as expulsions are continuing to occur even years after insertion. Experience in the technique of GyneFix insertion appears to reduce the incidence of late expulsion.

The expulsion rate was found to be slightly higher amongst nulliparous women than parous, although the difference was not statistically significant. It is not possible from this study to say whether the expulsion rate in our clients is lower than that observed with framed devices, as framed device users were not studied. The results of the Chinese study ${ }^{4}$ confirm that the expulsion rate of the GyneFix is lower than that of the T380A in parous Chinese women, but to date there has been no published randomised comparative study in nulliparous women. This is needed to assess whether expulsion is also less likely with the frameless device in this group of women. The commonlyheld belief that IUD expulsion rate is generally higher in nulliparous women than parous is not well substantiated in recent literature, and lower rates have been observed by some workers. ${ }^{7-10}$

Expulsion rates similar to ours have been observed in family planning clinics elsewhere in the UK (personal communication). In the majority of sites offering GyneFix in the UK, ultrasound scanning after insertion is not routinely performed unless the clinician has doubts about correct placement. The possibility of expulsion should be discussed with the client prior to insertion, along with a recommendation that she check for the presence of the thread after each menstrual period. Unnoticed expulsion should be confirmed by ultrasound and plain X-ray of the pelvis and abdomen, to ensure that the device has not perforated. Ongoing experience in the technique of GyneFix insertion is essential to retain expertise. Audit enables continued monitoring of expulsion rates.

Since the completion of the study described here, the insertion instrument has been substantially changed, in an attempt to simplify the insertion technique. This may result in fewer expulsions, particularly those occurring soon after insertion.

In spite of the expulsions, the majority of our users are satisfied with the GyneFix and we continue to receive many requests for insertion. We feel that the GyneFix is a welcome addition to the contraceptive menu offered to our clients.

\section{Conclusion}

The GyneFix expulsion rate in our service is higher than quoted in clinical trials. Expulsion appears unrelated to the parity of the user. Early expulsions may be related to insertion technique, representing insufficient implantation of the anchoring knot into the fundal myometrium. Late expulsions also occur, often many months after insertion; the reason for these is unclear. Users should be taught to check for the presence of the thread after each menstrual period and unnoticed expulsion should be confirmed by ultrasound and abdomino-pelvic plain X-ray.

\footnotetext{
Statements on funding and competing interests

Funding: None.

Competing interests: The authors work at the Abacus Centres for Contraception and Reproductive Health. In lieu of payment for training other clinicians in the technique of GyneFix insertion, Abacus has received some devices free of charge from Contrel, the manufacturer.

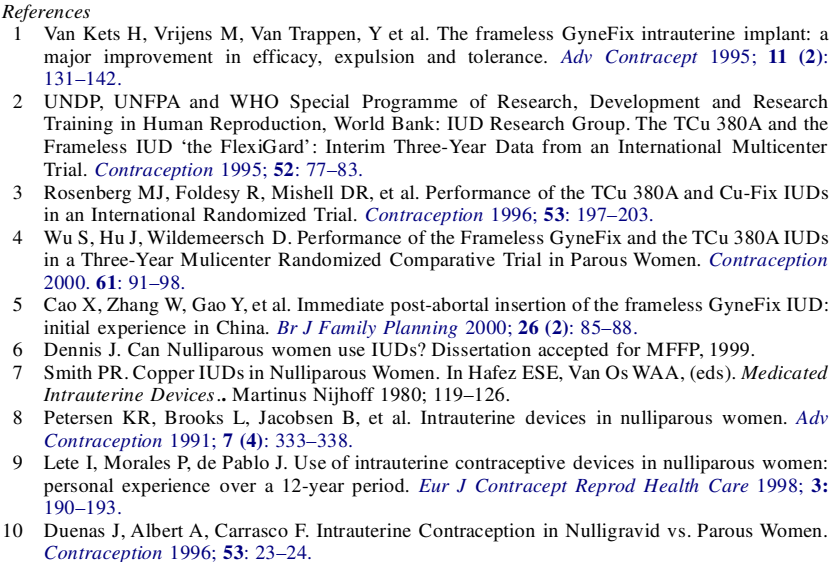

1 Van Kets H, Vrijens M, Van Trappen, $\mathrm{Y}$ et al. The frameless GyneFix intrauterine implant: a major improvement in efficacy, expulsion and tolerance. Adv Contracept 1995; 11 (2): major im $131-142$.

2 UNDP, UNFPA and WHO Special Programme of Research, Development and Research Training in Human Reproduction, World Bank: IUD Research Group. The TCu 380A and the Frameless IUD 'the FlexiGard': Interim Three-Year Data from an International Multicenter Trial. Contraception 1995; 52: 77-83.
Roser

Rosenberg MJ, Fesy R, Mishell DR, et al. Performance of the TCu 380A and Cu-Fix IUD an International Randomized Trial. Contraception 1996; 53: 197-203.

Wu S, Hu J, Wildemeersch D. Performance of the Frameless GyneFix and the TCu 380A IUD in a Three-Year Mulicenter Randomized Comparative Trial in Parous Women. Contraception

Cao X, Zhang W, Gao Y, et al. Immediate post-abortal insertion of the frameless GyneFix IUD: initial experience in China. Br J Family Planning 2000; 26 (2): 85-88.

6 Dennis J. Can Nulliparous women use IUDs? Dissertation accepted for MFFP, 1999.

Smith PR. Copper IUDs in Nulliparous Women. In Hafez ESE, Van Os WAA, (eds). Medicated Intrauterine Devices.. Martinus Nijhoff 1980; 119-126.

Petersen KR, Brooks L, Jacobsen B, et al. Intrauterine devices in nulliparous women. $A d v$ Contraception 1991; 7 (4): 333-338.

2 Lete I, Morales P, de Pablo J. Use of intrauterine contraceptive devices in nulliparous women personal experience over a 12-year period. Eur J Contracept Reprod Health Care 1998; 3 personal experience over a 12-year period. Eur J Contracept Reprod Health Care 1998; 3 ;
190-193.

Duenas J, Albert A, Carrasco F. Intrauterine Contraception in Nulligravid vs. Parous Women. Contraception 1996; 53: 23-24. 